\title{
Structure and Composition of Nanoscopic Domains in Functional Perovskite-Type Materials
}

\author{
Anke Weidenkaff ${ }^{b}$, Myriam H. Aguirre ${ }^{b}$, Thomas Lippert ${ }^{c}$, Uwe Falke ${ }^{d}$, and Ursel Bangert ${ }^{\star a}$
}

\begin{abstract}
A- and B-site substituted cobaltate perovskites $\left(\mathrm{ABO}_{3}\right)$ were prepared by soft chemistry and Pulsed Laser Deposition (PLD) processes. The formation of nano- and microdomains was examined by means of transmission electron microscopy to determine the influence on the materials properties. The growth of thin epitaxial films on oxide substrates by pulsed reactive cross beam laser ablation proceeds through island formation in StranskiKrastanov fashion; after the islands reach a certain height they develop laterally to form a dense epitaxial film. Orientation relationships, interfacial strain and surface roughness depend on the misfit with the respective substrate. Oxygen deficient La/Ca cobaltates exhibit Brownmillerite and Ruddleston-Popper defects, and increasing O-deficiency results in increasing resistivity of the films. Local defects are found as well in the microstructure of $\mathrm{Mn}$-doped cobaltates obtained by soft chemistry and sintered for long times at high temperatures. This perovskite phase exists in the orthorhombic phase at higher Mn-concentration and presents an array of inter-grown twin domains. Thin films of cobaltates with nominal composition of $\mathrm{La}_{0.6} \mathrm{Ca}_{0.4} \mathrm{CoO}_{3}$ exhibit catalytic activity for oxygen reduction and evolution in alkaline electrolytes. Control over the crystallinity of the thin film, achieved by pulsed reactive crossed beam laser ablation, was used to show that the catalytic activity varies with crystallinity. Single crystalline films exhibit the highest activity, followed by polycrystalline film and amorphous films. Even the orientation of single crystalline films has an influence on the catalytic activity.
\end{abstract}

Keywords: Catalyst · Defects $\cdot$ EELS $\cdot$ HRTEM $\cdot$ Perovskite

\section{Introduction}

Perovskite-type metal oxides are being used in an increasing number of applications, owing to their catalytic, electrical and optical properties [1][2]. More specifically the $\mathrm{ABO}_{3}$-type oxide, with $\mathrm{A}=\mathrm{La}, \mathrm{Ca}$ and $\mathrm{B}=\mathrm{Co}$ has become known as an electro catalyst in rechargeable metal/air batteries [3], and is also a candidate for thermoelec-

\footnotetext{
${ }^{\star}$ Correspondence: Dr. U. Bangert ${ }^{\mathrm{a}}$

aschool of Materials

The University of Manchester

Manchester M1 7HS, UK

Tel.: +44161306 3587

Fax: +44161306 3586

E-Mail: ursel.bangert@manchester.ac.uk

bSolid State Chemistry and Catalysis

Empa- Materials Science \& Technology

Überlandstrasse 129

$\mathrm{CH}-8600$ Dübendorf

cMaterials Group

Paul Scherrer Institut

$\mathrm{CH}-5232$ Villingen

dSuperSTEM Laboratory

CCLRC
}

Daresbury WA4 4AD, UK tric applications, in particular upon doping of the B-site with Ti or Mn [4-7].

Optimisation of these materials with respect to their functionality is difficult due to interaction with, e.g. electrode components, such as the carbon support. Hence, ideally perovskites on inactive substrates with, e.g. well defined electrolyte/oxide or electrical contact/oxide interfaces would be desirable. Furthermore, in order to study the bulk transport properties of functional perovskite-type phases without the effects of undefined grain boundaries, model systems are required; in contrast to pressed and sintered powders these would consist of single crystalline samples [8]. Single crystal perovskites can be obtained via epitaxial growth on various substrates. Inactive substrates like magnesium oxides, strontium titanates and lanthanum aluminates are suitable candidates. The presence of defects, in particular planar defects and twin domains, can be expected to have an impact on the functional performance. In this article we investigate the microstructural and stoichiometric integrity of rare earth/alkaline earth cobaltates and manganates, and relate the microstructural findings to electric transport properties. The study involves detailed investigation of planar defects via atomicscale structural and chemical analysis, for which leading edge high resolution electron microscopes (HRTEM), including the new generation dedicated, aberration corrected scanning transmission electron microscopes (STEM), equipped with energy dispersive analytical tools, e.g. electron energy loss spectroscopy, are utilised. Initial growth stages of the epitaxial perovskite films on single crystalline substrates are studied further with atomic force microscopy (AFM).

\section{Preparation of the Perovskite Films}

Crystal growth of large single crystals with e.g. the floating zone method is limited to congruently melting product phases. Many phases could not be produced with the floating zone furnace and with flux crystal growth attempts because of phase segregation processes during crystal growth of the complex phases with more than two different cations at high process temperature. Furthermore the anionic composition is not easy to control which makes it impossible 
to vary the anionic composition in most of the cases.

Epitaxial thin films grown on inactive substrate materials offer a suitable alternative to large single crystals as 'two-dimensional' crystals and the possibility to study the influence of the crystallographic orientation on the catalytic activity.

Thin films can be prepared by various techniques that range from chemical solution deposition (CSD) by e.g. sol-gel processes [9], over chemical vapour deposition (CVD) of e.g. metal-organic precursors, and physical vapour deposition (PVD) by e.g. RF sputtering and pulsed laser deposition (PLD). In the PLD process a target material is produced from the pressed powder sintered perovskite-type metal oxide phases. These targets are mounted inside the PLD chamber and rotated. With a laser beam part of the material is then ablated to form an ablation plume, i.e. a plasma containing the components of the perovskite phase in a highly excited state. This plasma travels in direction of the heated substrate to be homogeneously deposited on the rotating target. PLD is a most promising method for the deposition of high-quality thin films of materials with complex composition which cannot be obtained by other methods since they are thermodynamically unstable. Since PLD is a physical approach, by varying the ablation and deposition parameters the composition can be controlled to a larger extend compared to other synthesis methods.

A variation of PLD, pulsed reactive crossed-beam laser ablation (PRCLA), where a synchronized reactive gas pulse interacts close to the target with the ablation plumes, gives further control to optimize the film growth (general scheme shown in Fig. 1) [10][11]. The PLD parameters directly influence the film growth and surface quality of the films [12-14].

The ablation parameters that can be varied for this purpose are the laser energy, wavelength, pulse length and repetition rate, as well as the background pressure. These parameters control the state and kinetic energy of the ablated species. With the modified Pulsed Laser Deposition system reactive gases can be pulsed into the ablation plume. A $\mathrm{KrF}$ excimer laser $(\lambda=248$ $\mathrm{nm}$ ) with a pulse duration of $17 \mathrm{~ns}$ was used, and the rotating target was located approximately $5 \mathrm{~cm}$ from the substrate, but this distance can be varied. The target was ablated with a laser fluence of $7.6 \mathrm{Jcm}^{-2}$ at a repetition rate of $10 \mathrm{~Hz}$

Two different oxygen sources were used during film growth, i.e. a synchronized pulsed valve operating at a backing pressure of 1-3 bar of $\mathrm{O}_{2}$ or $\mathrm{N}_{2} \mathrm{O}(99.999 \%$ purity, pulse length of $400 \mu \mathrm{s}$ ) and a leak valve to provide an additional background pressure of $\mathrm{O}_{2}$ of $8 \times 10^{-2} \mathrm{~Pa}$ during the

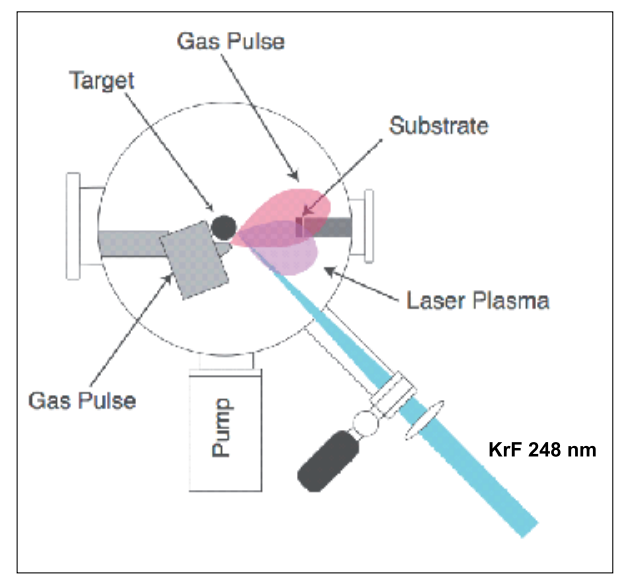

Fig. 1. Schematics of the pulsed reactive crossed-beam laser ablation (PRCLA) setup. A synchronized reactive gas pulse interacts close to the target with the ablation plumes. The plasma species are deposited on a rotated heated substrate.

deposition. The films were cooled with a cooling rate of $\approx 40{ }^{\circ} \mathrm{C} / \mathrm{min}$.

With the laser ablation of the oxide targets only a fraction of the oxygen is released as neutral atoms and ions, while the remaining part is ejected as $\mathrm{O}_{2}$. The adsorption probability of $\mathrm{O}_{2}$ on the substrate surface is lower than that of other plasma components [10][15]. Additional oxidizing sources for the effective oxidation of the cations during the film growth were applied to control the oxygen content. The application of the reactive gas pulse results in a brighter and larger ablation plume for all the applied perovskite-type phases. Dinitrogen oxide $\left(\mathrm{N}_{2} \mathrm{O}\right)$ is a strong oxidizing agent, which decomposes upon collisional fragmentation by photons from the plasma to form nitrogen and oxygen. With synchronized $\mathrm{N}_{2} \mathrm{O}$ gas pulses and a $8 \times 10^{-4}$ mbar oxygen background enough oxygen excess is created, arriving simultaneously with the species from the perovskite target at the substrate. It could be shown that the oxygen content of the films was enhanced when $\mathrm{N}_{2} \mathrm{O}$ instead of oxygen was used as oxidising agent in the gas pulse.

The films were grown on silicon $(\mathrm{Si})$, stainless steel, $\mathrm{MgO}$ (100), (110), (111), strontium titanate (STO) and lanthanum aluminate $(\mathrm{LAO})$ substrates $(10 \times 10 \times 0.5$ $\mathrm{mm}^{3}$ ) with polished surfaces heated to a defined temperature $\left(200^{\circ} \mathrm{C}<\mathrm{T}<850{ }^{\circ} \mathrm{C}\right)$. The single crystalline oxide substrates revealed a specified roughness of 1-2 $\mathrm{nm}$. The substrates were rotated during the deposition to obtain uniform film thicknesses.

With a defined number of laser/gas pulses the thickness of the deposited films can be varied from monolayer deposition to thick coatings of several micrometer thicknesses. The initial film formation was studied by growing films of different thicknesses with pulse numbers $5,10,20,30,70$, $200,250,500,750$ and 20000, resulting in a range of films from between less than $4 \mathrm{~nm}$ and $20 \mathrm{~nm}$ thickness, exhibiting nanoscopic islands, up to films of $200 \mathrm{~nm}$ thickness.

All samples prepared with 5-750 laser pulses were optically transparent, while films prepared with 20000 laser pulses were black and 'mirror-like'.

\section{Transmission Electron Microscopy}

Local microstructure, interfaces and film-substrate epitaxial relationship were investigated by cross-sectional and planview transmission electron microscopy. The structure and local microstructure of the powder perovskites were also investigated by HRTEM and diffraction. In the case of bulk materials, TEM samples were produced by cutting the $\mathrm{LCC} / \mathrm{MgO}, \mathrm{LCC} /$ $\mathrm{LaAlO}_{3}$ and $\mathrm{LCC}_{\mathrm{SrTiO}}$ samples along the [100] direction. These slices were glued by joining the LCC surfaces together. Thinning of the slices was performed using a combination of the 'tri-pod technique' (mechanical grinding, polishing) and Ar-ion milling. For powder perovskite compounds, a $\mathrm{Cu}$-grid was prepared with a few drops of the powder suspension in ethanol. Phase contrast or bright field (BF) high resolution electron microscopy (HREM) images were obtained in Jeol JEM 3000 EX with field emission gun. The lattice resolution Z-contrast or high angle annular dark field (HAADF) images and the electron energy loss (EEL) spectra were obtained in an aberration corrected [16] dedicated, cold-field emission scanning transmission electron microscope (STEM), the Daresbury SuperSTEM, equipped with a UHV Enfina EEL spectrometer. The aberration correction enables attainment of a $1-\AA$ probe, so that lattice images with sub- $\AA$ resolution and atomic column resolution EEL spectra can be obtained.

\section{A-Site Substitution in Cobaltates $\left(\mathrm{La}_{0,6} \mathrm{Ca}_{0,4} \mathrm{CoO}_{3-\delta}\right)$}

\subsection{Morphology}

Initially after a few pulses a first deposition of LCC takes place on the $\mathrm{MgO}$ surface. Atomic force microscopic (AFM) and electron microscopic (EM) studies on the initial growth steps reveal a 3D growth mode forming 10-20 nm diameter perovskite-type islands.

After further deposition with 70-500 laser pulses the height of the LCC deposit did not change, however, the lateral size of the grains increased up to a grain size of $100 \mathrm{~nm}$ in diameter due to coalescence of the 'LCC islands'. TEM studies of the films grown with 200 pulses show complete coverage of the substrate with - typical for a Stranski- 
Krastanov growth mechanism - additional islands of approximately $20-80 \mathrm{~nm}$ in diameter. The deposition after 750-20 000 laser pulses revealed dense films of 20-200 nm thickness.

Two-dimensional moiré fringes magnify the structural aspect and illustrate the relation between the film and the substrate. The TEM image of the plan-view specimen in Fig. 2 shows moiré fringes arising from the misfit between the cubic $\mathrm{MgO}$ and LCC crystals.

The moiré fringe pattern of the planview samples originated from the interference between a pair of electron beams. One beam is generated in the LCC and the second beam in the $\mathrm{MgO}$. Each reflection in the LCC acts as incident beam for the $\mathrm{MgO}$ and produces a double reflection which can be seen in the ED pattern of Fig. 2.

By measuring the spacing of the parallel moiré patterns we found a lattice spacing $\mathrm{d}=0.190 \mathrm{~nm}$ for the $(200)_{\mathrm{LCC}}$, which corresponds well with the theoretical value of $\mathrm{d}=0.191 \mathrm{~nm}$ confirming that the $(200)_{\mathrm{LCC}}$ planes are parallel to the $(200)_{\mathrm{MgO}}$ planes. The spacing of the moiré fringes (variation in the $(002)_{\mathrm{LCC}} \|$ to the $(002)_{\mathrm{MgO}}$ contrast) differs somewhat from one domain to another due to a slight tilt of the grains. Some areas without moiré fringes are visible because of the island structure of the LCC film.

The surface of the samples was examined by a Parc Scientific Autoprobe $\mathrm{CP}$ Atomic Force Microscope in tapping and in contact mode. The topographic 3D (AFM) image in Fig. 3 shows the shape of the islands. AFM studies of the films after LCC deposition reveal the different stages of island coalescence to form larger grains (see Fig. 3a). The results of the line profile analysis (Fig. 3b) expose a standard roughness of $28.3 \mathrm{~nm}$ and a lateral grain diameter of $100 \mathrm{~nm}$ to $900 \mathrm{~nm}$. Grain growth and nucleation are competing processes which lead to a slightly inhomogeneous size distribution. After the islands reach a height of approximately 10-20 nm they develop laterally to form a dense epitaxial film.

While layer by layer growth is always expected for homoepitaxial systems, the lattice mismatch between the substrate and LCC film in this heteroepitaxial system results in an initially 3D growth mode [17].

\subsection{General Microstructure}

The high-resolution TEM image in cross sectional view, shown in Fig. 4, represents the sharp interface of the $\mathrm{MgO}$ substrate (lower part) and a dense highly crystalline $\mathrm{La}_{0.6} \mathrm{Ca}_{0.4} \mathrm{CoO}_{3}$ film (upper part). The stress during the initial deposition process in film and substrate - caused by the impinging activated species on the substrate surface and the strain caused by the $10 \%$ lattice misfit (+ thermal misfit) between $\mathrm{MgO}$ and LCC - is partially relaxed. This results in an enhanced roughness of the sub-

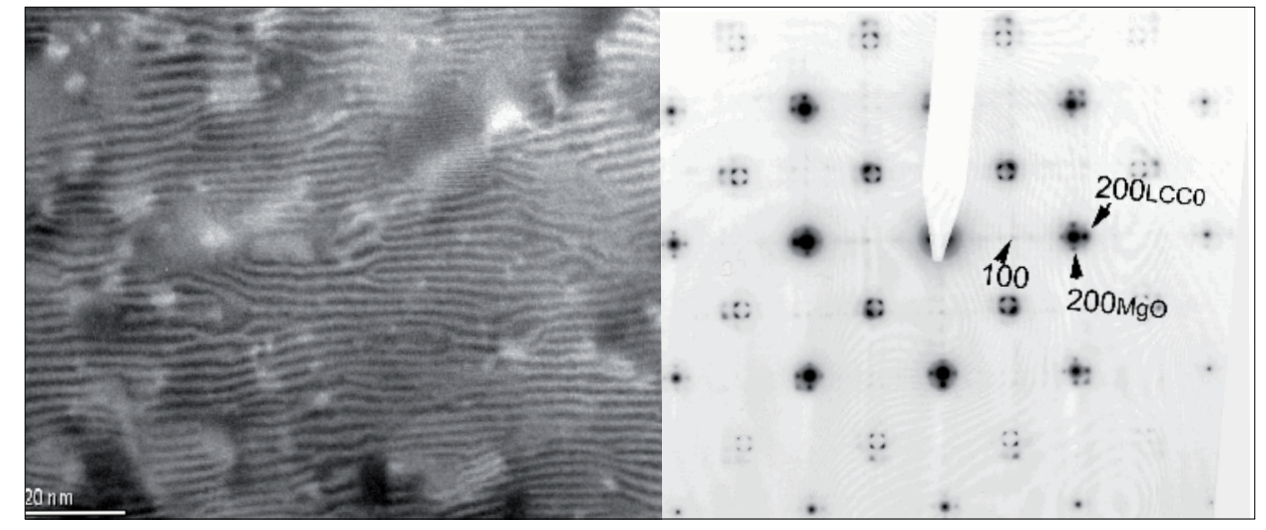

Fig. 2. TEM plan-view (a) of the LCC film on MgO showing the moiré fringes from the MgO and LCC film/nano-islands with (b) the corresponding double electron diffraction pattern strate at the interface and in the formation of a strained interlayer with defects.

The electron diffraction (ED) pattern reveals a cubic cell with $\mathrm{a}=0.380 \mathrm{~nm}$, which is comparable to values of the stress free LCC powder $(\mathrm{a}=0.3790 \mathrm{~nm})$ and shows furthermore that the $(002)_{\mathrm{LCC}}$ planes are oriented parallel to the $(002)_{\mathrm{MgO}}$ planes.

The lattice mismatch of the heteroepitaxy varies with the substrate material, i.e. $\mathrm{MgO}$ with $\mathrm{a}=0.421 \mathrm{~nm}$, STO with $\mathrm{a}=$ $0.3905 \mathrm{~nm}$ or LAO with $\mathrm{a}=0.3788 \mathrm{~nm}$.

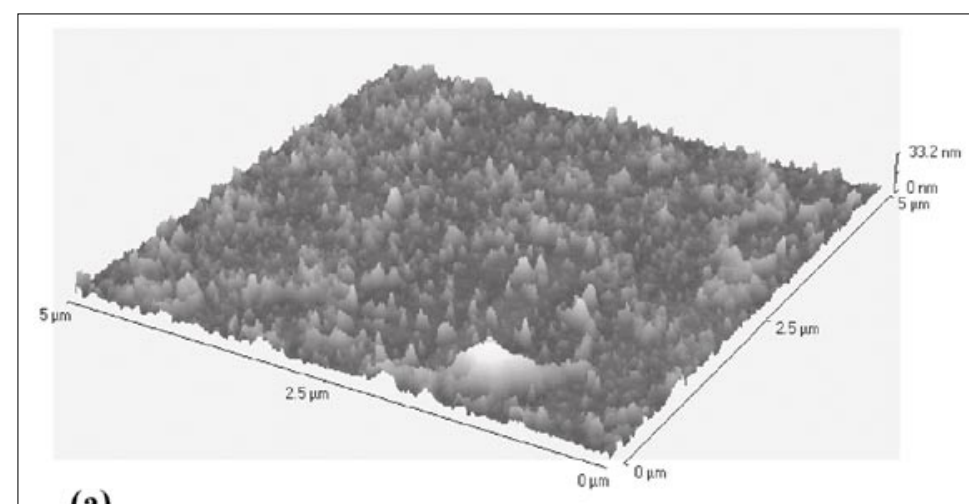

(a)
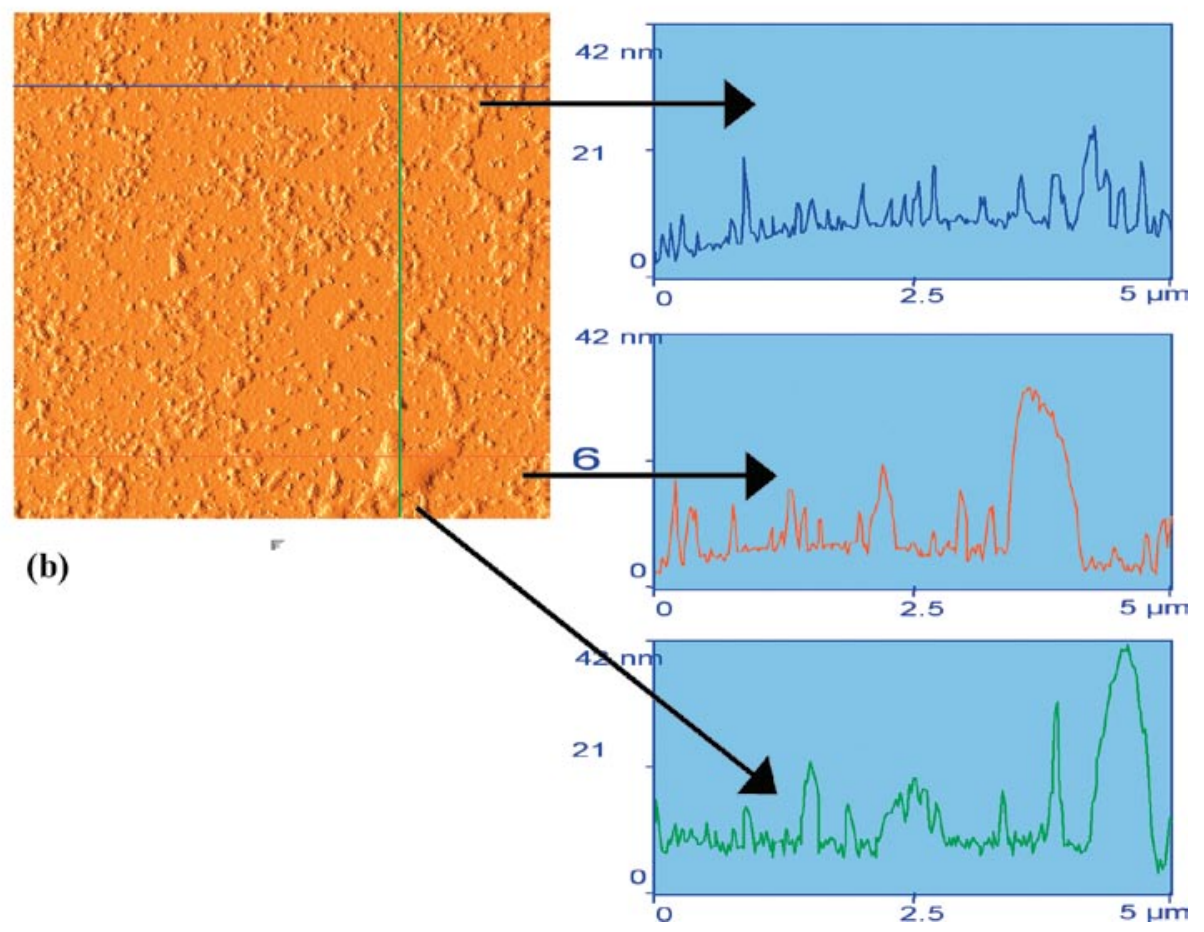

Fig. 3. AFM study of the LCC deposition after 750 laser pulses, showing (a) relief with (b) features up to $40 \mathrm{~nm}$ in height due to coagulating islands (less than 100 islands per square micrometer) 


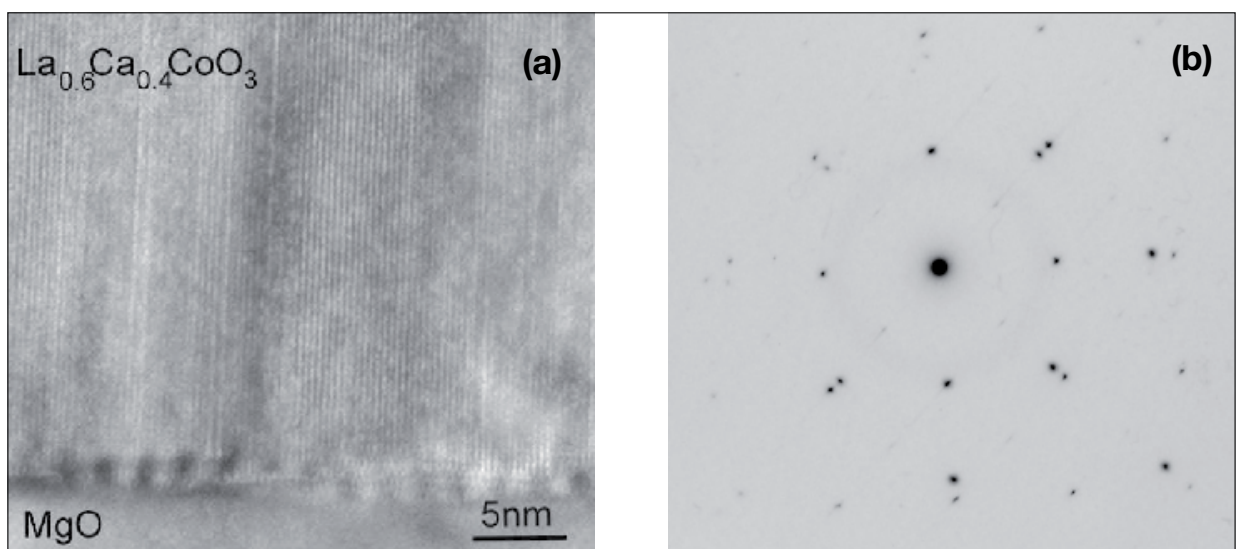

Fig. 4. HRTEM image of the LCC film/MgO interface (a) and electron diffraction of the LCC film on $\mathrm{MgO}(001)(\mathrm{b})$

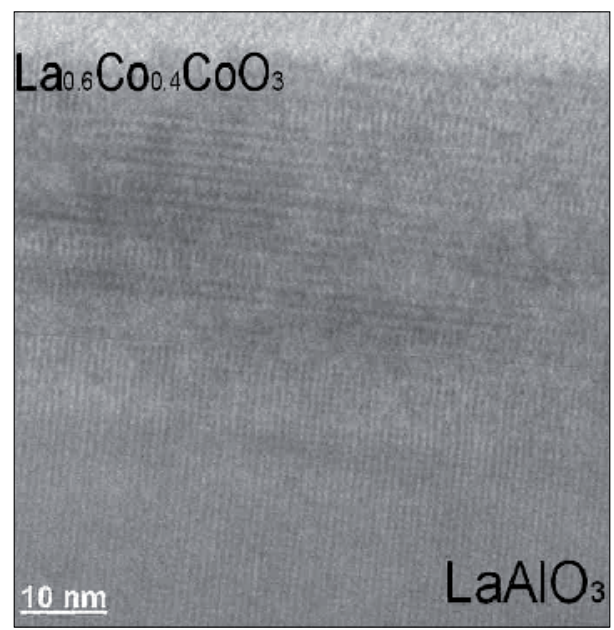

Fig. 5. Cross-sectional TEM-view of the LCCfilm/ LAO interface

Fig. 5 shows the interface of LCC with the LAO substrate. The mismatch is much smaller, and accordingly there is hardly any strain noticeable in the interface.

Films larger then $200 \mathrm{~nm}$ in thickness are generally black and mirror-like. Results of Rutherford backscattering (RBS) analysis of the elemental film composition confirm that the ablation is congruent.

XRD characterisation of $200 \mathrm{~nm}$ $\mathrm{La}_{0.6} \mathrm{Ca}_{0.4} \mathrm{CoO}_{3}$ films on $\mathrm{MgO}$ substrates reveals epitaxial growth of the perovskitetype metal oxide. The (200) ${ }_{\mathrm{LCC}}$ reflection of the perovskite phase, in addition to a

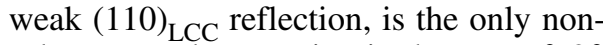
substrate peak appearing in the XRD $\theta-2 \theta$ scan at $\chi=0$. Therefore, it can be concluded that the $\mathrm{La}_{0.6} \mathrm{Ca}_{0.4} \mathrm{CoO}_{3}$ grows preferentially along the $\mathrm{MgO}$ (200) direction.

Fig. 6 shows the (111) pole figure of the LCC film at the $2 \theta$ angle of $42.97^{\circ}$. In this figure four maxima at $\chi=54^{\circ}$ are visible, indicative of the epitaxial single crystalline cubic domains of the orientated film.

The electrical resistivity of the films was measured with a four contact method as a function of temperature and oxygen, i.e. $\mathrm{Co}^{4+}$ content of the films [18] in a Quantum Design Physical Properties Measure-

ment System (PPMS). All cobaltate films show semiconductor behaviour. The resistivity of perovskites with similar cationic composition, but different oxygen content can vary by orders of magnitude (see Fig. 7). The increased $\mathrm{Co}^{4+}$ content results in an increased number of charge carriers [6][18]. Comparison of films with identical cationic stoichiometry and varying oxygen content shows that their behaviour follows the prediction. Comparisons of measurements on materials with high oxygen deficiency

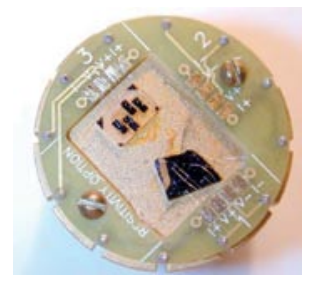

(a) different composition show an enhanced resistivity of up to 0.01 $\Omega \mathrm{cm}$ at room temperature.

\subsection{Brownmillerite Phases}

Oxygen-deficient LCC phases were prepared for cross-sectional HRTEM studies in order to study the formation of characteristic micro-heterogeneous domains in the nonstoichiometric perovskite-type oxides [19][20]. In HRTEM narrow domains of only a few unit cell widths can be observed, which proceed parallel to the basal plane in oxygen deficient crystalline $\mathrm{La}_{0.6} \mathrm{Ca}_{0.4} \mathrm{CoO}_{3}$ perovskite samples. This observation was similar for PLD thin films as well as for particles with the same composition. Fig. 8 shows a HRTEM crosssectional image of such domains, which appear as bright stripes running along $<110>$ directions.

These domains were identified by electron diffraction (ED) studies as local superstructures and as reduced phases with brownmillerite structure [21-23]. Under even higher resolution direct observation of two-fold super structures within the Co-O planes can be made in these films. These structures are thought to arise from ordered oxygen vacancies. Fig. 9 shows an HAADF lattice image with four planes ex-

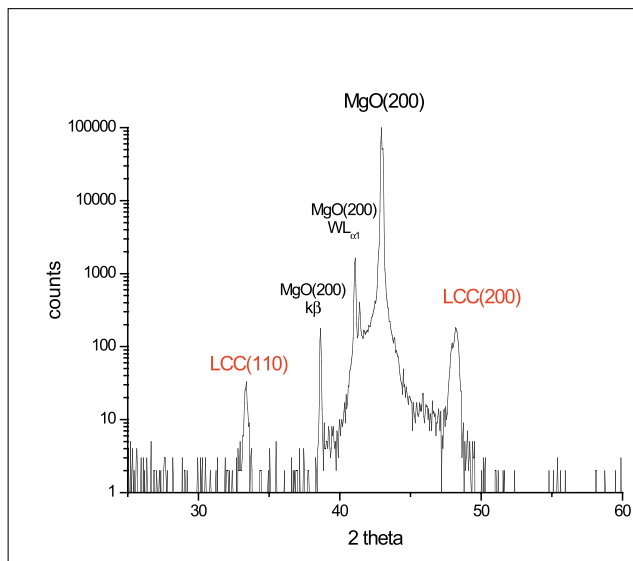

(a)

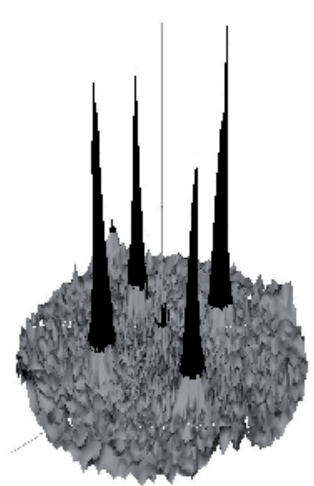

(b)
Fig. 6. XRD pattern (a) and 111 pole figure (b) of a $\mathrm{La}_{0,6} \mathrm{Ca}_{0,4} \mathrm{CoO}_{3}$ film grown on $\mathrm{MgO}$

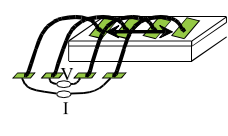

(b)

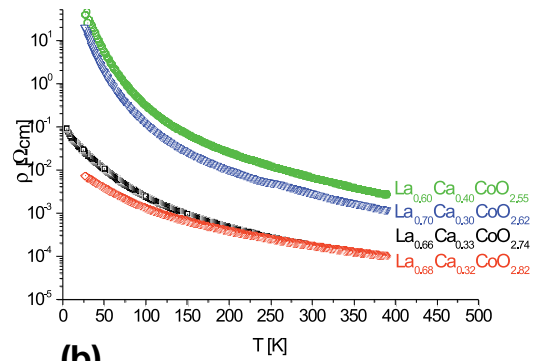

Fig. 7. (a) PPMS sample holder with contacted $10 \mathrm{~nm}$ thick (upper sample) and $200 \mathrm{~nm}$ thick (lower sample) contacted thin films and (b) results of four point measurements on $200 \mathrm{~nm}$ thick films with 

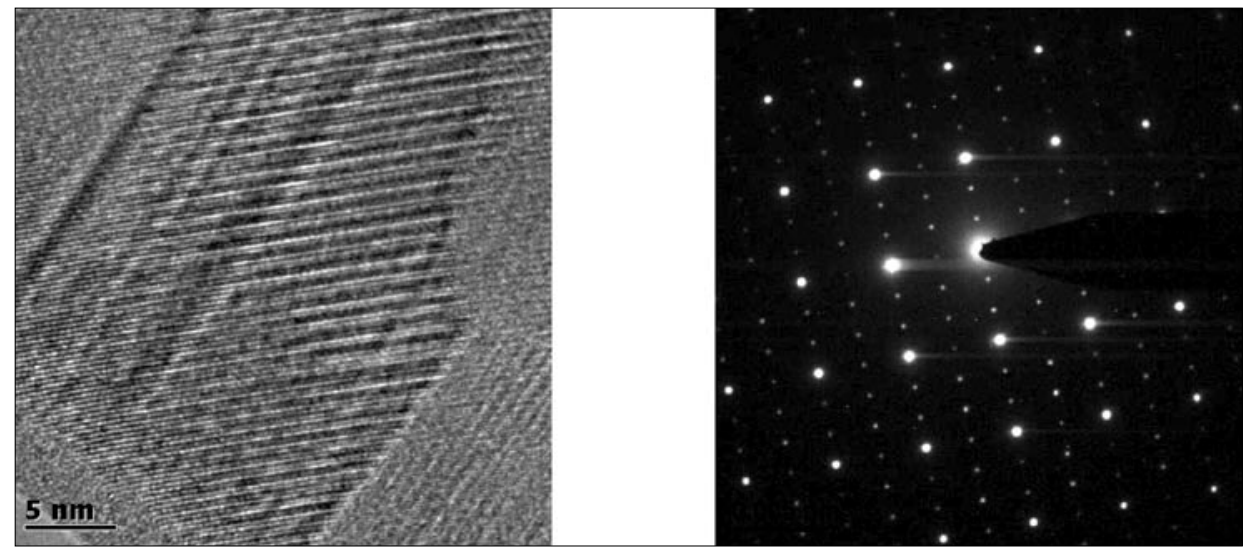

Fig. 8. TEM and $E D$ from domains in $\mathrm{La}_{0.6} \mathrm{Ca}_{0.4} \mathrm{CoO}_{2.7}$

hibiting these two-fold structures (arrowed), in which pairs of more closely spaced atomic columns (bright 'blobs') are separated by larger gaps. The contrast in atomic resolution HAADF Z-contrast images can be rather directly related to the atom positions, and it appears from inspection that $\mathrm{Co}$ atoms on sub-stoichiometric $\mathrm{Co}-\mathrm{O}$ planes form pairs as a result of repulsion due to an unscreened Coulomb potential. Oxygen vacancies would thus be located in the gaps between the pairs. These planes can be pictured as consisting of chains of corner sharing $\mathrm{CoO}_{4}$ tetrahedra. They are separated by perovskite-type layers with corner-sharing $\mathrm{CoO}_{6}$ octahedra. This arrangement resembles the brownmillerite-type structural model [24].

Overlaid on Fig. 9 (left hand top corner), is an intensity map of the backgroundsubtracted oxygen signal extracted from an electron energy loss spectroscopy line scan (similar to the line scan intensity profiles in Fig. 11). In locations where the EEL scan line crosses the two-fold structure the

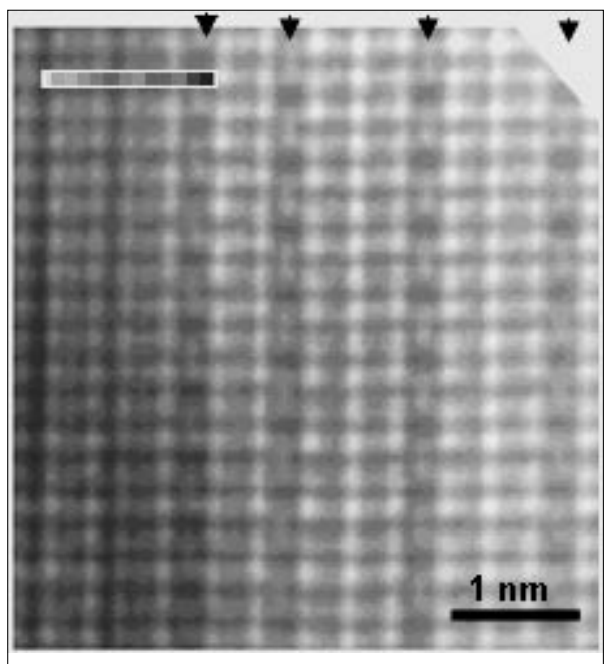

Fig. 9. Low-pass filtered HAADF STEM image showing vertical, planar, two-fold defects (arrowed), and also two RP defects on the left hand side. The grey-value strip in the left hand top corner shows the intensity of the O K-edge extracted from EEL spectra. contrast is black. This confirms the oxygen deficiency.

\subsection{Ruddelston-Popper (RP) Defects}

Another type of planar fault observed in LCC is the RP defect, and although the study of RP phases has a long history, this is the first time that its stoichiometry could be directly demonstrated via atomic resolution HAADF contrast in combination with modelling and atomic column-resolution EELS: in the LCC films Ca undergoes segregation into domains, where it is enriched by a factor of $\sim 2$ compared to the bulk. Fig. 10(a) shows HAADF Z-contrast of such domains, which occur at various separation distances. Fig. 10(b) shows the low-pass filtered section around the middle singular domain in the image of Fig. 10(a). The imthe planes horizontal and vertical. The contrast can be assigned to atomic columns: $\mathrm{La}$ with the highest $\mathrm{Z}$ and constituting $60 \%$ of the atoms in the column, clearly gives the brightest contrast in the HAADF image. not contribute much. Co has a lower $\mathrm{Z}$ than $\mathrm{La}$ and gives rise to the medium contrast values between the bright blobs, again, the $\mathrm{O}$ atoms in the same column do not contribute much to the contrast. The transition metal-atom plane is missing at the domain, resulting in two $\mathrm{La} / \mathrm{Ca}$ atom planes being next to each other. The atomic model with the suggested planar defect structure is overlaid on both images. The domains constitute $1 / 2$ unit-cell-wide rock salt lamellae. These are a result of missing Co-O planes, thus leading to two Ca-enriched layers lying adjacent and shifted by $<1 / 21 / 20>$ with respect to each other.

To prove the chemical nature of the planar defect, it was further investigated by EELS. Fig. 11 shows modelled profiles of the concentration ratio $\mathrm{Co} / \mathrm{La}$ for different domain stoichiometries along with experimental data (the scale is arbitrary). The experimental data were extracted from the age has been rotated by $\sim 40^{0} \mathrm{cw}$ to make The $\mathrm{Ca}$ and $\mathrm{O}$ atoms in the same column do
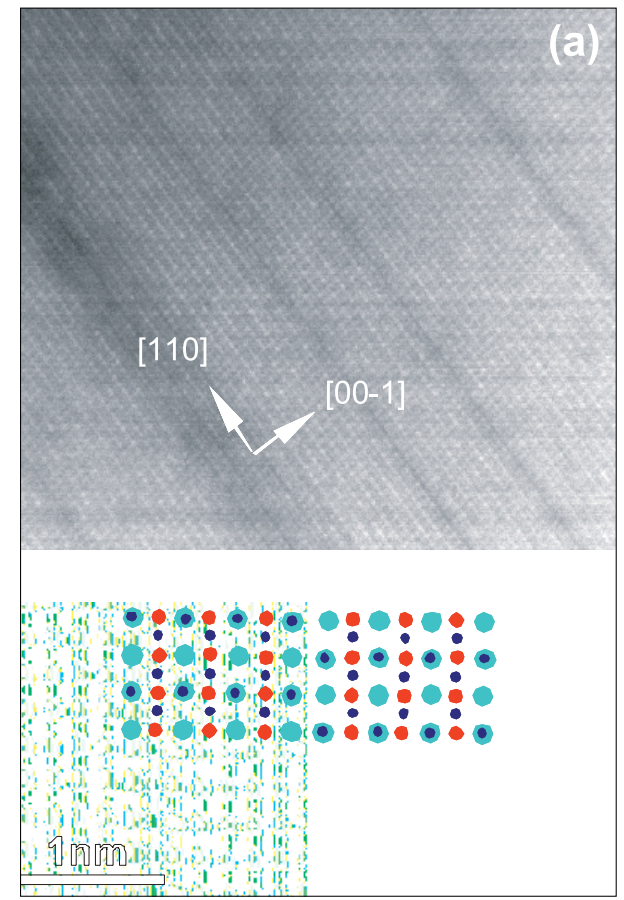

Fig. 10. Lamella-type features in an epitaxial $\mathrm{La}_{0.6} \mathrm{Ca}_{0.4} \mathrm{CoO}_{3}$ film on MgO; a) HAADF STEM image, b) part-filtered image with overlaid model structure. Turquoise: $\mathrm{La} / \mathrm{Ca}$, red: $\mathrm{Co}$, blue: $\mathrm{O}$.

respective absorption edges of spectra of an EEL line scan across the boundary, the location of which is set to zero. The periodic undulations reflect the course of the intensity as the beam probes over atomic columns and empty channels. The simulations show that the $\mathrm{Co} / \mathrm{La}$ ratio at the domain would be enhanced if the domain was pure $\mathrm{CaO}$. In this case no La atom would sit closer to the domain than the closest Co occupied sites, and thus the spatially broadened Co signal would overrule the La signal at the domain. In a domain with $\mathrm{La} / \mathrm{Ca}$ occupancy of the bulk stoichiometry $(x=0.4)$, there is a distinct drop in the Co/La ratio. However, the experimental results (grey curve) indicate a moderate drop. Also, whereas the Ca signal increases in absolute terms, the La-signal does not (curves not shown here). This can be explained by a significant increase, but not a complete substitution, by $\mathrm{Ca}$ at the domain. The measured and the modelled profile for $x=0.7$ have the highest correlation factor; hence an enrichment of $\mathrm{Ca}$ to $70 \%$ appears to occur at the domain. Similar values of the Ca-enrichment have been obtained from quantitation of the HAADF Z-contrast images via image simulation with multi slice (MS) calculations.

The nature of the domain could be further ascertained by analysis of the EEL spectra themselves; these were taken with a $1-\AA$ probe, which was channelled on an individual atomic column. Fig. 12 shows that when straddling the fault, the oxygen $\mathrm{K}$-edge fine structure resembles that of oxy- 


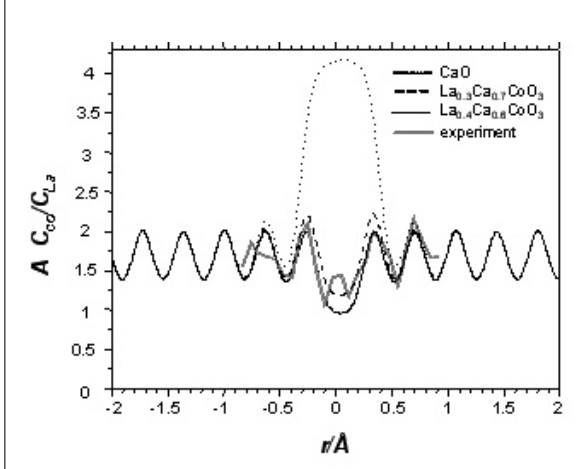

Fig. 11. Simulated and experimental Co/La intensity amplitude as a function of the distance from the domain, which is placed at zero, the bulk stoichiometry was assumed as $\mathrm{La}_{0.6} \mathrm{Ca}_{0.4} \mathrm{CoO}_{3}$ and $\mathrm{La} / \mathrm{Ca}$ ratios were varied at the planar defect

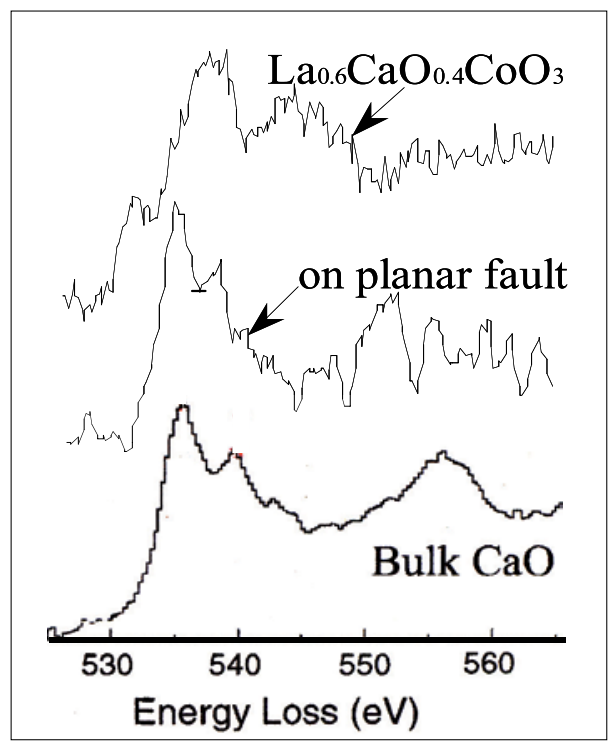

Fig. 12. EEL spectra, from top to bottom, taken in perfect perovskite regions, on the domain (difference spectrum) and of $\mathrm{CaO}$ bulk (courtesy $\mathrm{Gu}$ and Čeh [11]). The domain spectrum was obtained by subtracting a fraction of the perovskite bulk spectrum.

gen in the rock-salt configuration, whereas away from the boundary the typical of LCC O-edge fine structure occurs [25].

\subsection{Electrochemical Properties}

The electrochemical activity of the LCC films for the redox reactions was measured similar to the gas diffusion electrodes using a three-electrode arrangement with the LCC as a working electrode, a Pt-wire as counter-electrode, and an $\mathrm{Hg} / \mathrm{HgO}$ as reference electrode by means of a potentiometer [3]. The electrodes were dipped in a cell with a 1 molar solution of $\mathrm{KOH}$. Oxygen was pumped through the $\mathrm{KOH}$ solution for saturation. A potential was applied to the electrode, while the corresponding current was measured and normalized with respect to the electrode area (i.e. current density). The overpotential is the difference between

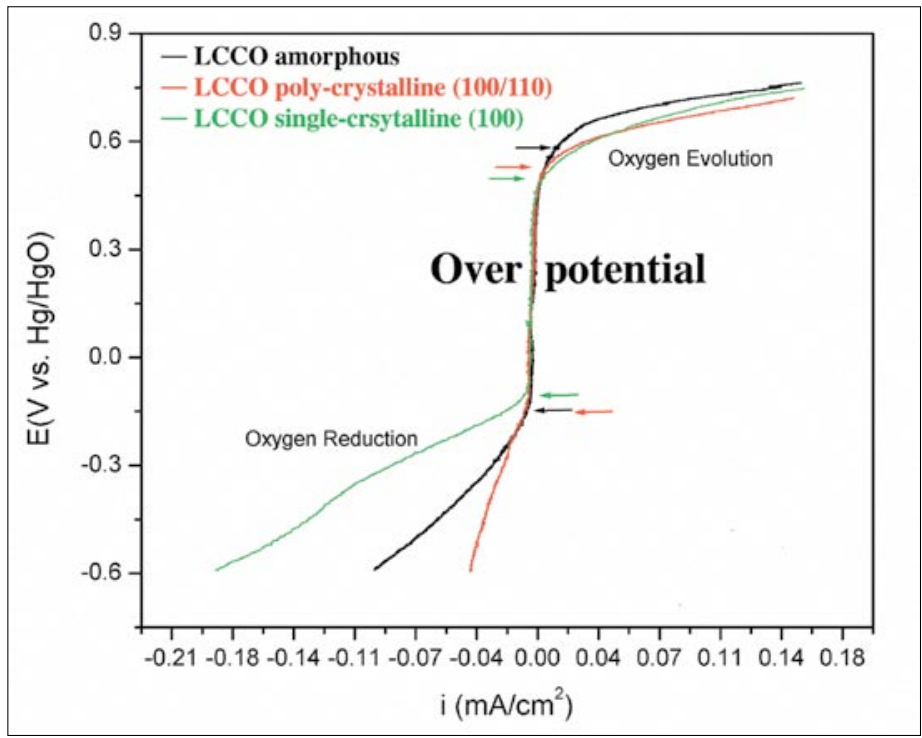

Fig. 13. Polarization curves of amorphous, single- and polycrystalline LCCO thin films deposited on $\mathrm{MgO}(100)$ substrates.

the values obtained for the two redox processes. The film with the smallest overpotential $(597 \mathrm{mV})$ is the $(100)$ oriented sample followed by the film with mixed (110) and (100) orientation $(679 \mathrm{mV})$ and the amorphous film $(738 \mathrm{mV})$. The polarization curves shown in Fig. 13 thus reveal that the crystallinity, i.e. crystallographic orientation, surface energy and grain boundaries, directly influences the catalytic activity for oxygen reduction and evolution reactions.

A comparison of single crystalline films with different orientation revealed that the catalytic activity is also influenced by the latter: higher activity (lower overpotential) was observed for films with (100) orientation compared to films with (111) orientation and films with mixed orientation $(100+$ 110) [13].

The films were analysed after several hours of redox processing and revealed no changes in structure and composition, which confirms the high redox stability of the LCC phase in alkaline media.

\section{B-Site Substitution in Cobaltates $\left(\mathrm{LaMn}_{1-\mathrm{x}} \mathrm{Co}_{\mathbf{x}} \mathrm{O}_{3-\delta}\right)$}

XRD and TEM studies on polycrystalline perovskite-type phases $\mathrm{LaMn}_{1-}$ ${ }_{\mathrm{x}} \mathrm{Co}_{\mathrm{x}} \mathrm{O}_{3-\delta}$ reveal that the Mn-rich compounds $\left(\mathrm{LaMn}_{1-\mathrm{x}} \mathrm{Co}_{\mathrm{x}} \mathrm{O}_{3+\delta}\right.$ with $x=0.05$ and 0.10 ) crystallise in the orthorhombic structure ( S.G. Pbnm), with cell parameters $\mathrm{a} \times \mathrm{b} \times \mathrm{c} \approx 5.53 \AA \times 5.58 \AA \times 7.79 \AA$ [7]. Electron microscopy, however, gave a more detailed view of the structure. The electron diffraction pattern simulated by fast Fourier transformation (FFT) from the image in Fig. 14 (where A- and B-FFT correspond to the $\mathrm{A}$ and $\mathrm{B}$ regions in the high-resolution image), shows a series of strong spots. In the case of A, they can be indexed on the basis of a simple perovskite cell $\left(a_{p} \sim\right.$ $3.9 \AA$, sub-index $\mathrm{p}$ => perovskite) viewed along the $[001]_{\mathrm{p}}$ axis. This is the substructure common to these types of materials [20]. Besides, it is possible to observe some spots indexed as $h / 2 \mathrm{k} / 20$, which suggest the presence of a so-called diagonal cell $a_{p} \sqrt{2} \times a_{p} \sqrt{2} \times 2 a_{p}$. This is characteristic of many perovskite structures or superstructures when there is an octahedral tilt. If the tilt is regularly distributed within the crystal, the corresponding maxima can be observed in X-ray or neutron diffraction; however, if this tilt does not exhibit long-range order, one can only see the diagonal cell by means of electron diffraction. Moreover, when the perovskite axial ratio is close to the ideal value of 2 , micro-domains are formed in which the long superstructure axis, in this case $c \approx 2 a_{p}$, is randomly distributed in one of the three space directions. The situation is clearly confirmed by the corresponding B-FFT appearing in Fig. 14. Micro-domains are observed in which the long $\mathrm{c}$-axis $(\mathrm{d} \approx$ $7.8 \AA$ A) alternates at random in different regions of the crystal, along the three space directions (Fig. 14 and 15).

Moreover, the Fourier transforms of each different A and B region in Fig. 15d are shown in Fig. 15a and 15b, respectively, while the FFT of the full micrograph (Fig. 15d) is given in Fig. 15c. Fig. 15c hence shows the global diffraction pattern; it is in fact the sum of the two patterns in Figs $15 \mathrm{a}$ and $15 \mathrm{~b}$. The high-resolution images on Figs $15 \mathrm{c}$ and 15d, enable us to properly interpret these patterns as showing the presence of three-dimensional inter-growth of micro-domains. It is possible to established the equivalent zone axes in the basic perovskite and orthorhombic structure as $\underset{\mathrm{s}}{\leftrightarrow}$ and $\leftrightarrow \leftrightarrow[1-10]_{\mathrm{o}}$, which are parallel (subscripts $\mathrm{p}$ and o mean perovskite basic cubic and orthorhombic cell, respectively).

The TEM studies confirm a highly crystalline and chemically homogeneous appearance for all compounds. No amor- 


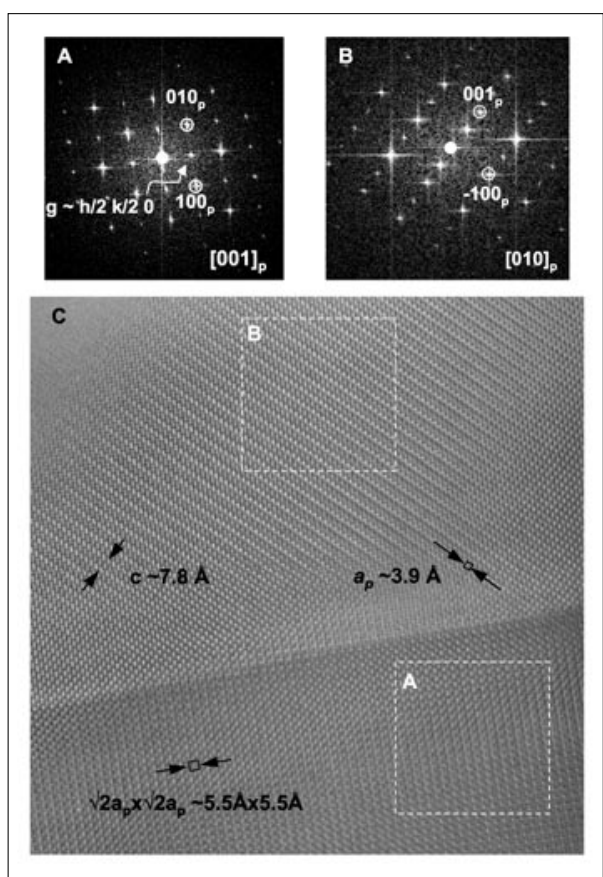

Fig. 14. FFTs obtained from boxes marked $A$ and $B$ in the high-resolution image marked $C$, representing the calculated diffraction patterns $A$ and $B$. These can be indexed on the basis of the perovskite subcell $a_{p} \sqrt{ } 2 \times a_{p} \sqrt{ } 2 \times 2 a_{p}$.

phous domains, crystalline impurities, defect, dislocation or secondary phases are observed.

\section{Conclusions}

In electrocatalytic reactions the processes on the surfaces as well as the electron and ionic transport in the bulk play an important role. These processes as well as the heat transport are influenced by the 'Realstruktur' of the transition metal oxide phases. The structure of domains formed in perovskite cobaltate and manganates (formed by means of A- and B-site doping) was studied via highly spatially resolved structural and chemical analysis using transmission electron microscopy. As key results it can be stated that O-deficient cobaltates contain Brownmillerite and Ruddleston-Popper defects, and exhibit increased resistivity. At high Mnconcentrations an orthorhombic perovskite phase occurs and presents an array of intergrown twin domains. The characterisation of the domains concerning crystal structure and composition is the prerequisite to understanding electrical transport properties and electrochemical performance.

\section{Acknowledgements}

The authors thank M. Montenegro for the preparation of the cobaltate films, C. Diecker for the preparation and measurements on the cs and pv samples as well as the Swiss Federal Office of Energy for financial support.

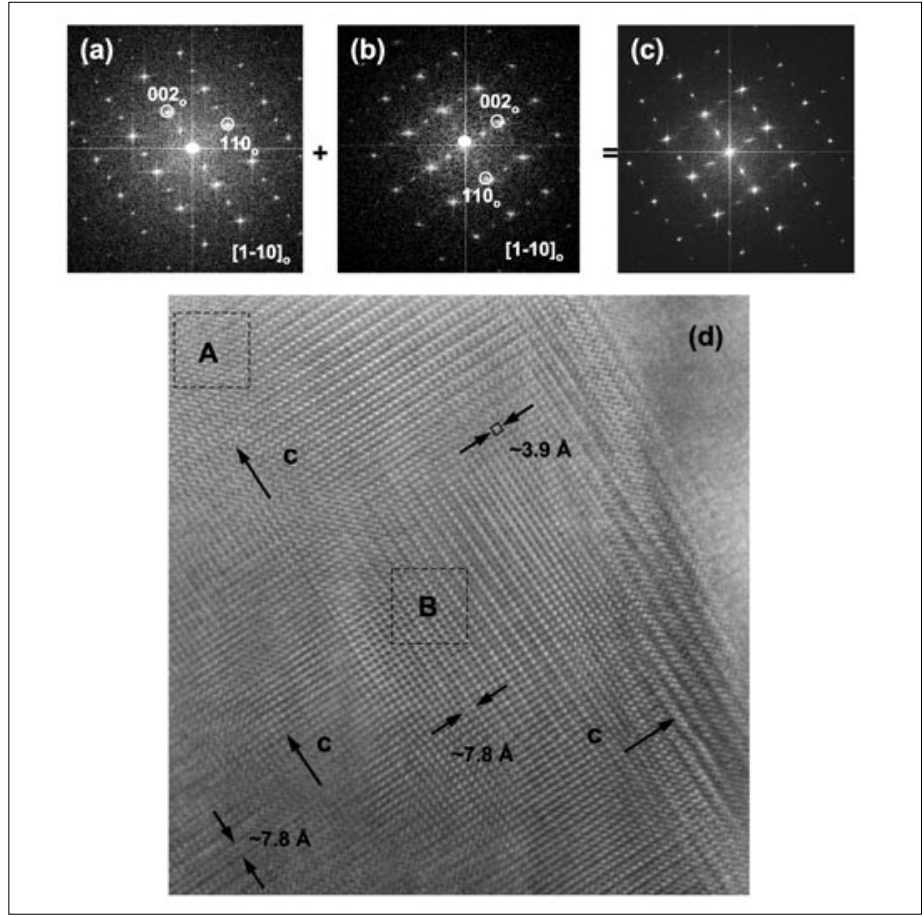

Fig. 15. a) Typical [1$10]_{0}=>[100]_{\mathrm{p}}$ and $\mathrm{b}$ ) $[1-10]_{0}=>[010]_{p}$ electron diffraction patterns, c) global pattern of the entire micrograph area below, d) HRTEM image of the inter-grown domains.

[1] F.S. Galasso, 'Perovskites and high Tc superconductors', Gordon and Breach Science Publishers, 1990.

[2] R.H. Mitchell, 'Perovskites Modern and Ancient', Almaz Press, 2002.

[3] S. Müller, K. Striebel, O. Haas, Electrochim. Acta 1994, 39, 1661-1668.

[4] A. Maignan, S. Hebert, L. Pi, D. Pelloquin, C. Martin, C. Michel, M. Hervieu, B. Raveau, Cryst. Engin. 2002, 5, 365-382.

[5] R. Robert, S. Romer, S. Hebert, A. Maignan, A. Reller, A. Weidenkaff, Proc. of the 2nd European Conference on Thermoelectrics, 2004, 194-197.

[6] R. Robert, S. Romer, A. Reller, A. Weidenkaff, Ad. Engin. Mater. 2005, 7, 303-308.

[7] L. Bocher, R. Robert, M.H. Aguirre, L. Schlapbach, Proceedings of the 4th European Conference on Thermoelectrics, 2006.

[8] M.J. Montenegro, M. Dobeli, T. Lippert, S. Muller, A. Weidenkaff, P.R. Willmott, A. Wokaun, Appl. Surf. Sci. 2005, 247, 197203.

[9] R. Aguiar, A. Weidenkaff, C.W. Schneider, D. Logvinovich, A. Reller, S. Ebbinghaus, Prog. Solid State Chem. in press.

[10] M.J. Montenegro, C. Clerc, T. Lippert, S. Muller, P.R. Willmott, A. Weidenkaff, A. Wokaun, Appl. Surf. Sci. 2003, 208, 4551.

[11] M.J. Montenegro, T. Lippert, S. Müller, A. Weidenkaff, P.R. Willmott, A. Wokaun, Phys.Chem.Chem.Phys. 2001, 4, 2799 2805.

[12] M.J. Montenegro, T. Dumont, T. Lippert, S. Müller, A. Weidenkaff, A. Wokaun, 'Pulsed Laser Deposition of thin oxide films: Applications in electrochemistry', in 'Pulsed Laser Deposition of Optoelectronic Films', INOE Publishing house, 2005, 2, 207-238.
[13] M.J. Montenegro, T. Lippert, S. Müller, A. Weidenkaff, A. Wokaun, 'Thin epitaxial oxide films as model systems for electrocatalysts', in 'Nanophotonics', Eds. H. Masuhara, S. Kawata, S., Elsevier, 2004, 251-273.

[14] S. Canulescu, T. Lippert, H. Grimmer, A. Wokaun, R. Robert, D. Logvinovich, M. Döbeli, A. Weidenkaff, Appl. Surf. Sci. 2006, 252, 4599-4603.

[15] M.J. Montenegro, T. Lippert, S. Müller, A Weidenkaff, P.R. Willmott, A. Wokaun, $A p$ pl. Surf. Sci. 2002, 198, 505-511.

[16] O.L. Krivanek, N. Delby, A.R. Lupini, $U l$ tramicroscopy 1999, 78, 1.

[17] A. Weidenkaff, C. Diecker, T. Lippert, M.J. Montenegro, Thin Solid Films 2004, 453, 406-410.

[18] T. Lippert, M.J. Montenegro, M. Döbeli, A. Weidenkaff, S. Müller, P.R. Willmott, A. Wokaun, Phys.Chem.Chem.Phys. in press.

[19] A. Nemudry, E.L. Goldberg, M. Aguirre, M.A. Alario-Franco, Solid State Sci. 2002, 4, 677-690.

[20] M.H. Aguirre, R. Ruiz-Bustos, M.A. Alario-Franco, J. Mat.Chem. 2003, 13, 11561160.

[21] A. Weidenkaff, S.G. Ebbinghaus, T. Lippert, M.J. Montenegro, C. Soltmann, R. Wessicken, Cryst. Eng. 2002, 5, 449-457.

[22] A. Reller, J.M. Thomas, D.A. Jefferson, M.K. Uppal, Proc. R. Soc. Lond. A 1984, 394, 223-241.

[23] S.Y. Istomin, O.A. Drozhzhin, G. Svensson, E.V. Antipov, Solid State Sci. 2004, 6, 539-546.

[24] O.H. Hansteen, H. Fjellvag, B.C. Hauback, J. Solid State Chem. 1998, 141, 411-417.

[25] U. Bangert, U. Falke, A. Weidenkaff, Mat. Sci.Eng. 2006, 133, 30-36. 